International Journal of Computer Networks \& Communications (IJCNC) Vol.7, No.4, July 2015

\title{
ANALYZING THE PERFORMANCE OF THE DYNAMIC POSITION LOCATION AND TRACKING (D-PL\&T) OF MOBILE NODES USING OMNI DIRECTIONAL ANTENNA IN MANET
}

\author{
Sharmistha Khan ${ }^{1}$, Golam R Khan ${ }^{2,}$ Dhadesugoor R. Vaman ${ }^{3}$, Siew T. Koay ${ }^{4}$, \\ Suxia Cui ${ }^{5}$ \\ ${ }^{1,2,4,5}$ Electrical and Computer Engineering Department, Prairie View A\&M University, \\ Prairie View, TX, USA \\ ${ }^{3}$ Texas A\&M University System Regents Professor (Retired),USA
}

\begin{abstract}
In this paper, we are focused to analyse the performance of the two dimensional dynamic Position Location and Tracking (PL\&T) of mobile nodes. The architecture of the dynamic PL\&T is developed based on determining the potential zone of the target node (s) and then tracking using the triangulation. We assume that the nodes are mobile and have one omnidirectional antenna per node. The network architecture under consideration is cluster based Mobile Ad Hoc Network (MANET) where at an instance of time, three nodes are used as reference nodes to track target node(s) using triangulation method. The novel approach in this PL\&T tracking method is the "a priori" identification of the zone of the target node(s) within a circle with a reasonable radios, and then placing the three reference nodes for the zone such that a good geometry is created between the reference nodes and the target nodes to improve the accuracy of triangulation method. The geometry of the reference nodes' triangle is closer to equilateral triangle and all potential target nodes are inside the circle. We establish the fact that when the target node is moving linearly, the predictive method of zone finding is sufficient to track the target node accurately. However, when the target node changes the direction, the predictive method of zone finding will fail and we need to place the three references outside the zone such that proper geometry with no one angle is less than 30 degrees is maintained to get accurate PL\&T location of the target node at each instance of time. The new zone is always formed for each instance of time prior to triangulation.

In this paper, we demonstrate the accuracy of integrated zone finding and triangulation for detecting the PL\&T location the node at each instance of time within 1.5 foot accuracy. It should be noted that as the target node is tracked continuously by applying the integrated zone finding and triangulation algorithm at different instances of time, one foot accuracy can no longer be maintained. Periodically, the good PL\&T data on each node has to be established by reinitializing the PL\&T locations of the nodes including those that are used as reference nodes. In this paper, the performance of the dynamic PL\&T system is derived using Additive White Gaussian Noise (AWGN) channel; and using AWGN plus Multi-path fading channel. The impact of multipath fading on tracking accuracy is analysed using Rician Fading channel for MANET applications outdoors. Our real time simulations show the PL\&T tracking accuracy for the mobile target nodes in both cases to be within 1.5 foot accuracy.
\end{abstract}

DOI : $10.5121 /$ ijcnc.2015.7401 
International Journal of Computer Networks \& Communications (IJCNC) Vol.7, No.4, July 2015

\section{KEYWORDS}

Dynamic Position, Localization, Tracking, Zone Prediction, Triangulation, Multipath Fading.

\section{INTRODUCTION}

Most of the research in wireless networks in the last three decades focused on increasing the data rate of transmission or spectral efficiency (bits/second/Hertz) and Bit Error Rate performance. It is only in the last few years, researchers are looking at optimizing the real time PL\&T tracking of mobile nodes for accurate determination of the target node at each instance of time. Of interest in this research is to use the pre-determined PL\&T location of the nodes within a cluster to provide optimal routing methods for multi-hop connectivity and for ensuring switching the connected path pre-emptively when a node is moving outside the realm of path connectivity for maintaining high probability of uninterrupted service provisioning. PL\&T location tracking is time domain alternate to measuring the received power at the destination node to find the distance between a source node and the destination node for optimizing the transmission power. Frequency domain methods of power measurements are often erroneous due to transmission impairments and noise.

Many researchers have addressed PL\&T methods in the last few years. Global Positioning System (GPS) is the most popular for tracking of any device in networks. However, it cannot provide the tracking accuracy when there is no line-of-sight path to the satellites that means it cannot work accurately in indoors or near to the buildings [1]. Other methods have also been developed including "measuring the received signal strength (RSS)", computing the "angle-of arrival (AOA"), "the finger printing", "time-of-arrival (TOA) measurement", and "timedifference-of-arrival (TDOA)" method [2]. The AOA-based techniques require an antenna array system that causes the hardware complexity and cost. The fingerprinting approach requires storing a large amount of radio characteristic information that is also increases complexity. In TOA based tracking methods rely on the propagation time of a signal travelling from transmitter to receiver. TDOA based tracking methods depend on processing the time (PT) difference of the multiple signals. Thus in both TOA and TDOA based tracking systems require good geometry for triangulation which is not often guaranteed when there is no prediction of where the target node is. They also require good accuracy of the clock with drift compensation for maintaining good synchronization between the nodes to ensure minimal errors in the tracking. However, the digital technology has progressed to the point where synchronization is not a significant problem these days. In this paper, we present the zone finding prior to the PL\&T triangulation to ensure placing the reference nodes to generate a good geometry to maintain accuracy of tracking. The triangulation requires three reference nodes to track a target node in two dimensional (X, Y) space and would require four reference nodes for tracking a target node in three dimensional (X, $\mathrm{Y}, \mathrm{Z})$ space. Since repeated triangulation of the same target would be susceptible to errors as the limits of errors can increase the tracking inaccuracy, it is critical that both the reference nodes and the target nodes to be reinitialized with a known location data over time for continuous tracking. In this paper, the performance of integrated zone prediction and 2D triangulation is derived through real time simulation in a MANET cluster, where the wireless AWGN channels and AWGN plus Fading channels have been used [3]. The results are summarized.

\section{BACKGROUND}

Different PL\&T methods have been developed to determine the location point of a target node accurately. Most researches used triangulation methods where single reference node, two reference nodes, and three reference nodes are used [4,5, and 6]. To analyse the performance of tracking accuracy, some of the important PL\&T methods are reviewed through literatures that are summarized in Table 1. 
International Journal of Computer Networks \& Communications (IJCNC) Vol.7, No.4, July 2015

Table1. Different Prediction Methods

\begin{tabular}{|c|c|c|}
\hline Prediction Methods & Features & Limitations \\
\hline $\begin{array}{l}\text { Forward Movement } \\
\text { based Prediction [7] }\end{array}$ & 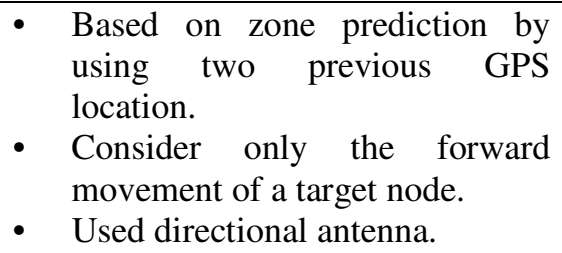 & $\begin{array}{l}\text { - Does not consider random } \\
\text { movement, sharp turns or } \\
\text { obstacles. } \\
\text { - GPS is not accessible in } \\
\text { indoor situation. }\end{array}$ \\
\hline $\begin{array}{l}\text { Location tracking } \\
\text { algorithm for } \\
\text { MANET using } \\
\text { directional antennas } \\
\text { with fixed beams [8] }\end{array}$ & $\begin{array}{l}\text { - } \text { Based on that transmitting node } \\
\text { indicates the need to switch the } \\
\text { antenna to an active antenna. } \\
\text { Transmitting node always } \\
\text { monitor the received power } \\
\text { level }\end{array}$ & 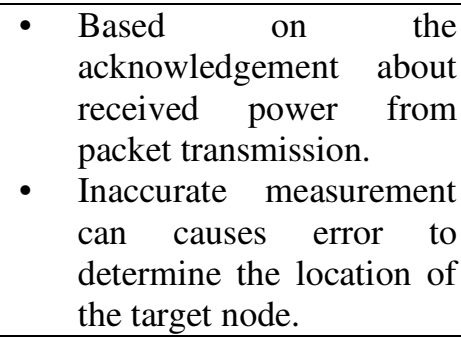 \\
\hline $\begin{array}{l}\text { Multi hop based } \\
\text { Prediction [9] }\end{array}$ & $\begin{array}{l}\text { - Used directional antenna } \\
\text { - Used a pair of reference nodes } \\
\text { and the angle of arrival (AoA) of } \\
\text { the best signal from each } \\
\text { reference node. } \\
\text { Using multiple levels of } \\
\text { reference nodes. }\end{array}$ & $\begin{array}{ll}\text { - } & \text { Increases cumulative } \\
\text { errors in multi hop } \\
\text { measurements. } \\
\text { - No beam adaptation used. } \\
\text { - Significantly reduces } \\
\text { accuracy of tracking. }\end{array}$ \\
\hline $\begin{array}{c}\text { Directional Lines } \\
\text { Intersection based } \\
\text { Prediction [10] }\end{array}$ & $\begin{array}{l}\text { - Sensor nodes estimate their } \\
\text { positions using the minimum } \\
\text { number of directional points. } \\
\text { Based on the intersection point } \\
\text { of the greatest gain direction of } \\
\text { directional antenna. } \\
\text { Cost effective and lower } \\
\text { computational complexity. }\end{array}$ & $\begin{array}{l}\text { - Does not address random } \\
\text { trajectory. }\end{array}$ \\
\hline $\begin{array}{l}\text { Distributed Position } \\
\text { Localization and } \\
\text { Tracking (DPLT) of } \\
\text { Malicious Nodes in } \\
\text { Cluster Based Mobile } \\
\text { Ad hoc Networks } \\
\text { (MANET) [6] }\end{array}$ & 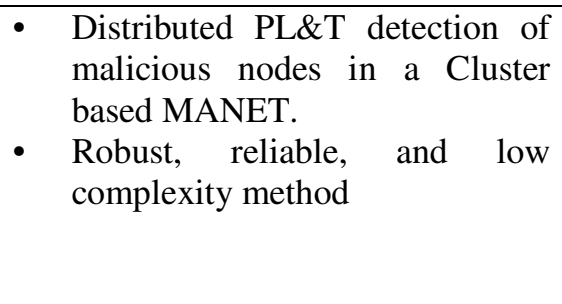 & $\begin{array}{l}\text { - Only forward movement is } \\
\text { considered for determining } \\
\text { the adaptive beam } \\
\text { formation. }\end{array}$ \\
\hline $\begin{array}{c}\text { DPL\&T using } \\
\text { Location based Hash } \\
\text { Scheme for Malicious } \\
\text { Detection under } \\
\text { Doppler Spread } \\
\text { Rayleigh Channel } \\
\text { [11] }\end{array}$ & $\begin{array}{l}\text { An integrated security and } \\
\text { dynamic PL\&T method. } \\
\text { - } \quad \text { Maintained two friendly nodes } \\
\text { Used KV technique for forward } \\
\text { error correction. Achieved } \\
\text { greater tracking accuracy using } \\
\text { lower Eb/No in fast Doppler } \\
\text { spread Rayleigh channel. }\end{array}$ & 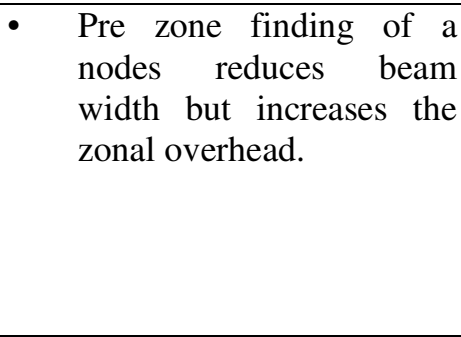 \\
\hline
\end{tabular}


By considering the major limitations of all the above methods, the integrated PL\&T method [3] is developed that uses IP based triangulation based on zone prediction method where accuracies of less than a meter are required. In this method, nodes require re-initializing after repeated tracking at different instances of time to maintain the tracking error within the specified target. The reference nodes also are changed dynamically as the target nodes move. Therefore, interchanging the reference nodes and target nodes was dynamically accomplished to maintain accuracy. Thus, this method may increase the overall complexity. On the other hand, multi-path fading, phase transition radiation and Doppler effects could play a significant role in reducing the accuracies of the signal [12]. Many researchers are addressing these issues through equalization, estimation and coding techniques [13-14]. Dynamic PL\&T is one of the most important application of resource efficient distributed mobile Adhoc network management system [15-16]. Some researchers use directional antenna which basically allows concentration of beam power in one direction in order to increase the signal strength and increase the probability of handling interferences and improve the PL\&T accuracy [17]. Researchers have also used Directional Antennas in interacting of steered or switched antenna systems in an ad hoc network [18]. However, the main limitation on using the directional antenna is the incapability on using for Multiple Input Multiple Output (MIMO) technology. Again, it is very difficult to use directional antenna in the end user's device. In recent years, in wireless communication field, for getting satisfactory performances, significant progress has been made in developing the overall systems that use multiple antennas at the transmitter and at the receiver [19] which known as multiple input multiple output (MIMO) systems. Therefore, Omnidirectional antenna is really beneficial for mobile and wireless devices those have MIMO technology as it can provide a 360 degree horizontal radiation pattern [20]. Considering this issue, omnidirectional antenna is used in this DPL\&T method.

\section{OVERVIEW OF DYNAMIC PL\&T SYSTEM}

The design of dynamic PL\&T system uses a sequential process of "zone finding" followed by "triangulation", where the "zone finding" allows locating the target node(s) and the triangulation computes the PL\&T location of the target node(s) accurately by placing the references to create reasonably perfect geometry [3]. The block diagram of the dynamic PL\&T system that involves several steps is shown in Fig. 1. Perfect geometry allows minimizing the outliers when the range is determined. In this design, we quantize the time such that the computational time for integrated zone finding and triangulation of a specific location is significantly smaller and allows the node to be virtually stationary for computation. The next location will be identified at a periodic quantized time. This process does not take into account the location in between two quantized times. It is anticipated that the importance of quantizing the time will allow computational accuracy and at the same time the transient period is not critical for the overall tracking of the mobile node. PL\&T is a continuous operation of finding the location of the target node with successive zone finding, placing the references for proper triangulation geometry and performing the triangulation in an atomic operation. The developed algorithm is able to find out the location of a target moving in any direction continuously. If a target node moves linearly in any one direction, it can be seen that the zone prediction method itself provides reasonably accurate prediction. However, the zone prediction can produce erroneous PL\&D data of the target when it does not move linearly. In [3], this has been shown using true path of the target node using a spiral. It also can be seen that the integrated zone finding and triangulation achieves significant accuracy in PL\&T prediction when the target moves in a spiral motion or any other non-linear motion. 


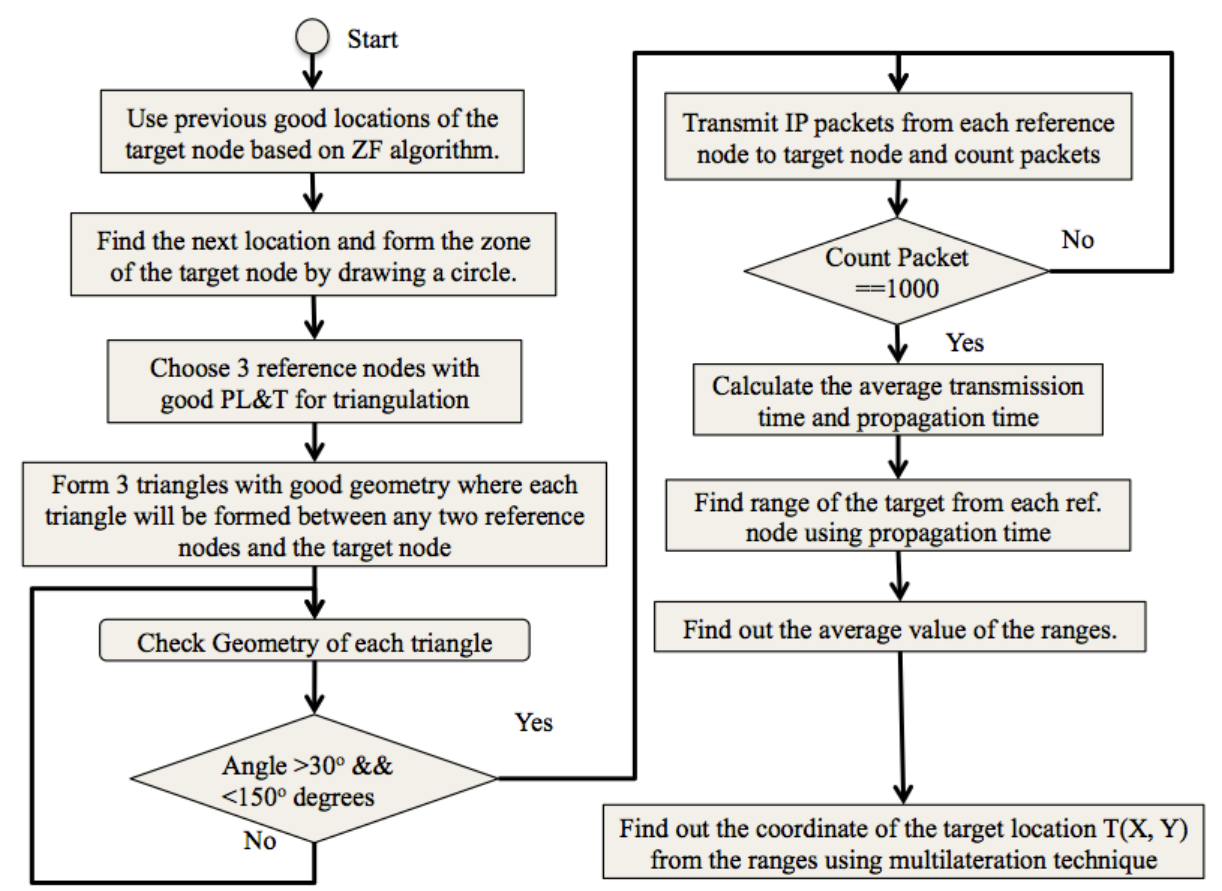

Figure 1: Block diagram of dynamic PL\&T architecture.

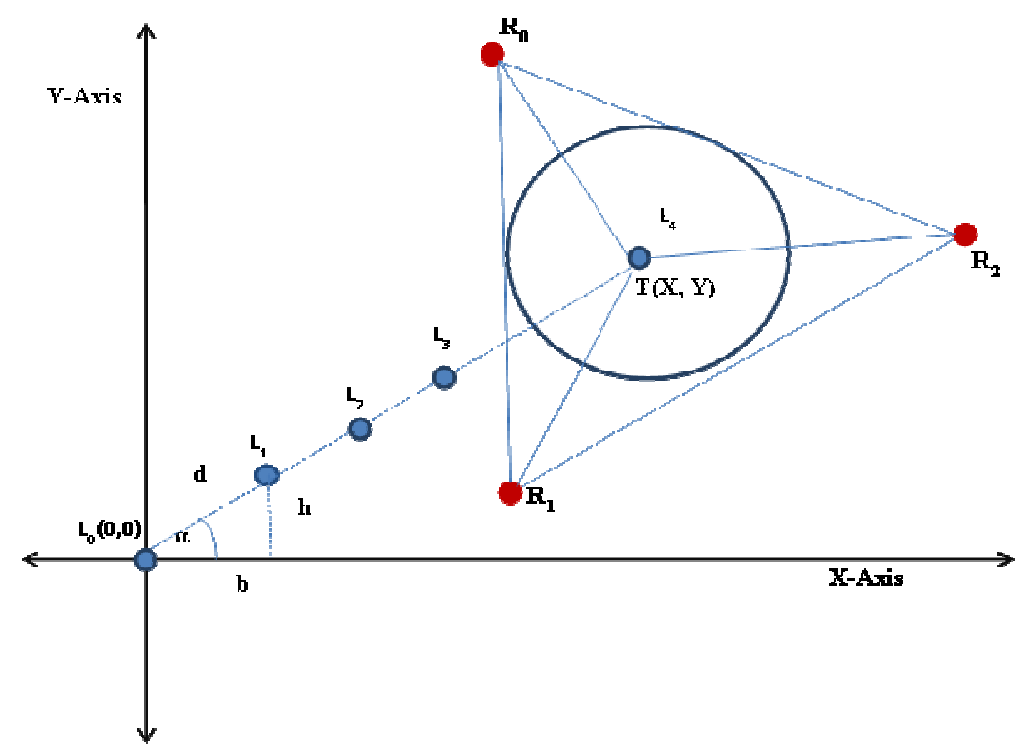

Figure 2: System Architecture of Zone Finder Algorithm.

Fig. 2 shows the basic design architecture of the dynamic PL\&T system where it is considered that the target node is moving linearly in a straight line with no change of direction. There are four nodes which are used as three references, R0, R1 and R2, and a target node at X, Y specified as $\mathrm{T}(\mathrm{X}, \mathrm{Y})$. L0, L1, L2, L3 shows the first four location points of the target node at earlier times. The circle around the target node is drawn with a radius equal to half the distance between the fourth and the fifth locations. The circle shows the predicted zone of the target node's location. 
The zone finding algorithm is used to find the zone of the fifth location based on the previous four good PL\&T locations. In fact, it is equivalent to predict the next location based on four previous good locations. This process is continuous for find the zone of the target node over time. Each time it is required to find out the predicted zone of the target node. In triangulation, after finding the zone, three reference nodes $(\mathrm{R} 0, \mathrm{R} 1, \mathrm{R} 2)$ has been placed, whose locations are known and form three triangles $\Delta \mathrm{R} 0 \mathrm{~T} \mathrm{R} 2, \Delta \mathrm{R} 0 \mathrm{~T} \mathrm{R} 1$, and $\Delta \mathrm{R} 1 \mathrm{~T} \mathrm{R} 2$, with the target node for performing triangulation process.

\subsection{Overview of Zone Finder (ZF) Algorithm}

The ZF algorithm uses a predictive polynomial of nth order where $n$ has to be at least 3. ZF algorithm computes the vector distance of the target from a reference point R1 (Fig. 2). The vector distance is defined by using equation (1). Reference point is basically a starting point since the polynomial is self-embedded [3].

$$
X(t)=\sum_{k=0}^{n} a_{k} t^{k} \ldots \ldots \ldots \ldots(1)
$$

Where $\mathrm{n}=$ order of the polynomial, $\mathrm{a}_{\mathrm{k}}$ is the coefficient of the $\mathrm{k}^{\text {th }}$ order of $\mathrm{t}$, and $\mathrm{t}$ is the time at which object is located. $\mathrm{X}(\mathrm{t})$ describes the trajectory of the moving object in time.

Where,

$$
d_{j}=\frac{\left|\prod_{\substack{i=0 \\ i \rightarrow j}}^{m-1}\left(t_{m}-t_{i}\right)\right|}{\left[\prod_{i=0}^{n}\left(t_{j}-t_{i}\right) \mid\right.} \ldots \ldots \ldots \ldots
$$

$d_{j}$ are the coefficients created for use in computing the vector distance $X(m)$ of the target from the reference point $\mathrm{R} 1$ in order to continuously track the object based on the $\mathrm{m}$ immediately preceding locations:

$$
X\left(t_{m}\right)=\sum_{k=0}^{m-1} d_{k} * X\left(t_{k}\right) \ldots \ldots \ldots
$$

To demonstrate the ZF algorithm, we make the following assumption in equation (1): $n=3 ; a_{3}=1$; $\mathrm{a}_{2}=-1 ; \mathrm{a}_{1}=2$ and $\mathrm{a}_{0}=5$. Then, we have got,

$$
\begin{gathered}
X(t)=t^{3}-t^{2}+2 t+5 \ldots \ldots \ldots(4) \\
d_{j}=\frac{\left[\prod_{i=0}^{3}\left(t_{4}-t_{i}\right) \mid\right.}{\left[\begin{array}{c}
i \neq j \\
\left.\prod_{i=0}^{3}\left(t_{j}-t_{i}\right)\right]
\end{array}\right] \ldots \ldots \ldots \ldots(5)} \\
X\left(t_{4}\right)=\sum_{k=0}^{3} d_{k} * X\left(t_{k}\right) \ldots \ldots \ldots(6)
\end{gathered}
$$

As long as the target node is going in linear motion, through this ZF algorithm, it is possible to predict the location point of the target node accurately. 


\subsubsection{Determination of Zone:}

By implementing the ZF algorithm, the distance between fourth and fifth location point can be found. The fifth location point is considered as the target location. To find out the co-ordinate of the fifth location point, it needs to assume some measurement that is shown in Fig. 2.It need to assume,

- The co-ordinate of first location point is $\mathrm{L}_{0}(0,0)$.

- Let, the first location point makes angel $\theta$ with the X Axis through its true path.

- From ZF algorithm (That is discussed above), the trajectory locations (the distance between one point to another point) of a node for an instance of time can be found. As the distances between two consecutive location points are obtained $\left(1^{\text {st }}\right.$ location point to $2^{\text {nd }}$ location point and $2^{\text {nd }}$ to $3^{\text {rd }}$ location point), by applying the trigonometric formula, the co-ordinate of the $2^{\text {nd }}$ point, $3^{\text {rd }}$ point, $4^{\text {th, }}$, and $5^{\text {th }}$ point accordingly can be computed.

The value of $\mathrm{X}$-axis and $\mathrm{Y}$-axis have found by using the following equation (7) - (10) where $\mathrm{b}$ is the coordinate value of $\mathrm{X}$-axis, $\mathrm{h}$ is the coordinate value of $\mathrm{Y}$-axis and $\mathrm{d}$ is the distance between two consecutive location points.

$$
\begin{gathered}
\cos \theta=\frac{b}{d} \ldots \ldots(7) \\
b=d \cos \theta \ldots \ldots(8) \\
\sin \theta=\frac{h}{d} \ldots \ldots \ldots(9) \\
h=d \sin \theta \ldots \ldots(10)
\end{gathered}
$$

After getting the co-ordinate of the $5^{\text {th }}$ location point, a circle is drawn around the $5^{\text {th }}$ point with a particular radius where the value of the radius will be the half distance between $4^{\text {th }}$ and $5^{\text {th }}$ point. Basically, the perimeter of this circle is considered as a zone for the target.

\subsubsection{Place Reference Point}

Three reference points have been placed those have the accurate PL\&T such as $\mathrm{R}_{0}\left(\mathrm{X}_{0}, \mathrm{Y}_{0}\right)$, $\mathrm{R}_{1}\left(\mathrm{X}_{1}, \mathrm{Y}_{1}\right), \mathrm{R}_{2}\left(\mathrm{X}_{2}, \mathrm{Y}_{2}\right)$ around the target node such a way that they will be outside of the zone and will form three triangles with good geometry for triangulation process.

Actually zone prediction method is fairly accurate for finding the PL\&T of a target node based on previous good location data when the target is moving linearly in one direction, it is not adequate when the nodes change directions. However, once the zone is predicted, the triangulation will provide accurate position of the target node by placing the reference nodes to form a reasonably perfect geometry.

\subsection{Triangulation after Zone Finding}

Basic principle of this method based on three points including two reference node points and a target node point [3]. Basically the triangles will be formed between any two reference nodes and the target node. Triangulation based PL\&T has drawback of the cumulative error during localization and tracking as nodes are continuously tracked at different locations [5]. 


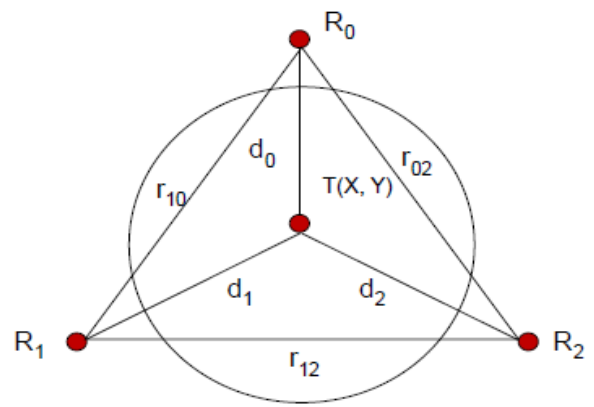

Figure 3. Triangulation with three reference nodes around the predicted zone

To get good geometry, it is required to carefully place the reference nodes such that there is no angle less than 30 degrees or greater than 150 degrees on the triangles.

\subsubsection{Determination of Range}

After forming the triangles with good geometry, the range between each reference node and the target node is determined. Fig. 3 shows the design of the triangulation with three reference nodes around the predicted zone where $R_{0}, R_{1}, R_{2}$ are three reference nodes and $T$ is the target node that form three triangles: $\Delta R_{0} T R_{2}, \Delta R_{0} T R_{2}$, and $\Delta R_{0} T R_{2}$. The ranges between the target node and the reference nodes will be found by exchanging the IP packets with time stamps of Time of Departure (ToD) and Time of Arrival (ToA). We have to keep record the values of ToA and ToD of each transmission of a packet to find out the transmission time which is the difference between ToA and ToD. N number of packets such as $\mathrm{P}_{1}, \mathrm{P}_{2}, \ldots, \mathrm{P}_{\mathrm{n}}$ will be send from one reference node to target node. For each packet transmission, it needs to record the time of departure (TOD) such as $t_{1} d_{0}, t_{2} d_{0, \ldots}, t_{n} d_{0}$ in the sender side. Again, on the receiving end it needs to record the time of arrival (TOA) such as $\mathrm{t}_{1} \mathrm{a}_{0}, \mathrm{t}_{2} \mathrm{a}_{0, \ldots, \ldots} \mathrm{t}_{\mathrm{n}} \mathrm{a}_{0}$. According to the TOD and TOA of each packet, the time difference between TOD and TOA for each packet can be computed. Then, the average transmission time is computed by equation (11).

$$
\text { Average Transmission Time }=\sum_{i=1}^{n} \frac{\left(T O A_{i}-T O D_{i}\right)}{n} \ldots \ldots \text { (11) }
$$

After finding the average transmission time, average propagation time is found by using the following equation (12).

Average Propagation Time $=$ Average Transmission Time- Average Processing Time

It needs to use a reference value as a propagation time that relates to an indexed range or distance. By this way, the range $d_{0}$ between the reference node $R_{0}$ and the target node $T$ has found. It needs to repeat the above process two times for getting the ranges $d_{1}$ and $d_{2}$ from other two reference nodes $R_{1}$ and $R_{2}$ to the target node $T$ respectively.

\subsubsection{Determination of X-Y Coordinates}

After getting all the ranges between each reference nodes to the target node, the average value of the ranges has found. Finally, by using the rages in multilateration technique, the coordinate of the target location $\mathrm{T}(\mathrm{X}, \mathrm{Y})$ have determined. As this algorithm will able to track the location of a target node continuously, it is require to repeat the overall procedure as well as the computation continuously for predicting the location of the target node in the network. 
International Journal of Computer Networks \& Communications (IJCNC) Vol.7, No.4, July 2015

\subsection{Use of Dynamic References}

For maintaining accurate processing of PL\&T using triangulation, it is critical that the nodes that are chosen to become "References", must maintain accurate PL\&T for themselves. When the "References" move, they lose the ability to maintain accurate PL\&T as PL\&T algorithm has a statistical accuracy. Periodically, these "References" are reverted to being "Targets" for PL\&T computations. The exchange of making the node either a "Target" or a "Reference" makes reference to be dynamically changed. As long as the "References" chosen have accurate PL\&T with the most recent measurements and they are placed after "zone finding" to ensure perfect geometry, the PL\&T computation will be maintained accurately. The statistical error at each location for the PL\&T measurement of a given target can become inaccurate over successive PL\&T measurement of that target over different times, due to cumulative errors being built during the PL\&T computations. Thus dynamic references are needed for applying proper geometry in triangulation method instead of stationary references.

\subsection{PLT under Multipath Fading}

Generally in wireless communications, fading refers to as the distortion of a signal transported on a channel [18]. The signal distortion occurs due to multipath. In a typical wireless communication environment, due to reflection, diffraction and scattering by different objects, multiple propagation paths often exist from a transmitter to a receiver. Copies of wireless signals following different paths can have different delays, random phases, attenuation and distortions. At the receiver, constructive and destructive interference may occur. When destructive interference occurs, the received signals can be very weak and many bit errors could occur. In wireless systems, the signal distortion that occurs due to the multipath propagation is referred to as multipath induced fading. There are many models exists that describe the phenomenon of small scale fading [21]. Out of these models, Rayleigh fading, Rician fading and Nakagami fading models are most commonly used to model a wireless channel. In this research, we have considered Rician Fading to analysis the effect of it's on tracking accuracy. The main focus of this research is on PL\&T of moving targets and it is not the intent of handling different fading channels. We only considered one model, "Rician", to demonstrate the effect of fading on the tracking accuracy. The use of other models is left for future research.

Rician fading model is commonly used in Line Of Sight (LOS) channels mostly outdoors. In Rician fading, there is a strong dominant component is present that is stationary. This dominant component is commonly known as the LOS (Line of Sight Component). Usually, Rician fading occurs when there is a NLOS (Non Line of Sight) along with LOS path present between a transmitter and a receiver [22]. A Rician fading channel can be described by two parameters: $\mathrm{K}$ and $\Omega$ where $\mathrm{K}$-factor is the ratio of signal power in direct path and the signal power in the other, scattered, paths. $\Omega$ is the total power from both paths, and acts as a scaling factor to the distribution [58]. For Rician Fading channel, $\mathrm{K}$ factor can be in between 1-12 where for Rayleigh fading $\mathrm{K}$ will be $=0$ (-infinity $\mathrm{dB}$ ) [23-24].

\subsubsection{Impact of Fading}

In this research, we have analyzed the impact of fading on the accuracy of our developed PL\&T method. We have added Rician fading channel where the value of Rician fading factor is used as $\mathrm{k}=6$. We need to find out the standard deviation of packet transmitted for triangulation process under AWGN channel and under fading channel to analyze the impact of fading. To find out the standard deviation, first we have to find out the average or mean of the transmission delay for an ensemble of packets. Then, we have to calculate the differences between mean and the 
transmission delay of each packet. Finally, we have to compute the standard deviation (sigma or $\sigma)$ by using following equation (13).

$$
\sigma=\sqrt{\frac{1}{N}}\left[\left(p_{1}-\mu\right)^{2}+\left(p_{2}-\mu\right)^{2}+\left(p_{3}-\mu\right)^{2}+\cdots+\left(p_{N}-\mu\right)^{2}\right] \ldots \ldots
$$

Where,

$$
\begin{aligned}
& \mu=\text { mean }=\frac{1}{N}\left(p_{1}+p_{2}+\cdots+p_{N}\right) \\
& p_{i}=\text { Transmissiondelay for } i^{\text {th }} \text { packet, } \\
& N=\text { masmher of narkets }
\end{aligned}
$$

To analyze the effect of fading, we have to perform the triangulation process under Rician fading channel for some ensembles of packets. For each ensemble of packets, we have to calculate the average or mean of the transmission delay and standard deviation of the transmission delay. Usually fading increases the transmission delay. Therefore, when we will see that the standard deviation of the transmission delay will be higher than the threshold value, it means the performance of the transmission is affected due to the fading channel. To find out the PL\&T of a target node accurately under a fading channel, we need to do the triangulation when fading goes away. Thus, it requires the standard deviation of the transmission delay under fading need to be below the value of standard deviation of transmission delay under AWGN channel. Therefore, when we will find the value of the standard deviation of the transmission delay under fading channel is higher than the threshold value, we have to ignore the transmission delay of that ensemble of packets to find the range as well as the coordinate of the target node of that instance of time. On that time, we have to use previous good location data to find out the location of the target node. We have already discussed the method of triangulation for finding the coordinate of a target node in section 3.2. By following the same procedure, we have to find the coordinate of a target node under a fading channel.

\section{SIMULATION METHODOLOGY}

To analyze the performance of the dynamic PL\&T method, we have run a real time simulation where Network Simulator-2 (NS2) [43] is used as a simulation tool for performing triangulation with zone finding and MATLAB is used for computing several measurements and for plotting different graphs from simulation results . This real time PL\&T system is deployed in MANET of a cluster size of $1000 \times 1000$ sq. m. terrain area using 40 nodes with random way point mobility. Since, this PL\&T system is an integrated method of "Zone Finding" and "Triangulation", first, we have run the simulation for Zone Finding method and then, we have performed the simulation for Triangulation using the predicted zone of a target node that we have determined from Zone Finding method. Finally, the integrated PL\&T method is used to determine the exact X-Y coordinates of the location point of any target nodes at any instance of time in the cluster accurately. The simulation is executed both under AWGN channel and under multipath faded Rician channel of frequency $2.54 \mathrm{GHz}$. In a cluster, we have considered two basic types of random motion of a node.

Linear Motion: In linear motion, we assume that the target node traverses in one direction linearly and does not change its direction while in motion. It implies that the target node moves linearly along a straight line.

Spiral Motion: Here, we consider the movement of the target to have continuous change of direction by creating a true path of the target using a "spiral graphic way". In addition, we will create a "trapezoidal graphic way" that has both linear path as well as abrupt change of direction at the end of each linear path. 
International Journal of Computer Networks \& Communications (IJCNC) Vol.7, No.4, July 2015

We have simulated these two particular cases for tracking the position location of a target node. For both cases, we have simulated the zone finding (ZF) algorithm and triangulation method to track the position location of a target node for its seven location points.

\subsection{Simulation Parameters}

We have listed some important simulation parameter in Table 2.

Table 2: List of simulation parameters

\begin{tabular}{ll}
\hline Parameters & Values \\
\hline Number of Packets & 1000 \\
Packet Size & 512 bytes \\
Area & $1000 \mathrm{~m} \times 1000 \mathrm{~m}$ \\
Antenna Type & Omnidirectional \\
Antenna Coordinate & $\mathrm{X}=0 ; \mathrm{Y}=0 ; \mathrm{Z}=1.5 \mathrm{~m}$ \\
Antenna Gain & $\mathrm{Gt}=1.0 ; \mathrm{Gr}=1$ \\
Transmitting Power & $0.2818 \mathrm{~W}$ \\
Frequency & $2.4 \mathrm{e}+9$ \\
Carrier Sense Threshold & $1.559 \mathrm{e}-11$ \\
Receiver sensitivity Threshold & $3.652 \mathrm{e}-10 ; \# 250 \mathrm{~m}$ \\
Rician Fading Factor & $1-8$ \\
\hline
\end{tabular}

\subsection{Simulation of PL\&T under Wireless channel}

\subsubsection{PL\&T of Target Nodes for Linear Motion}

When a target node will be in linear motion, to track the position location of that target, the simulation has done for triangulation algorithm under both AWGN channel and Rician Fading Channel. As the zone finding algorithm is based on polynomial function, multipath fading has really no impact on finding the zone. We have determines the zone of the target by following the procedure that we have discussed in section.

\section{Simulation Result of Triangulation after the Zone Finding:}

Once the locations within the zones are identified, the triangulation process is executed to find the exact location. We have already described the triangulation process. We use this process of triangulation algorithm to find the exact locations of a target. We have used NS-2 as a simulation tool for performing the simulation of triangulation process that consists of several steps. Initially, we have run the simulation under AWGN channel and then we have run the simulation under the Rician Fading Channel, where the fading model is used that is incorporated in NS2. In this simulation, we have found the locations of a target for 7 instance of time for a particular example that we have discussed in section 3.1. Every time when a target node changes its location, it also dynamically changes all reference nodes' position by satisfying all the condition for making good geometry on triangulation. By applying equation (11), we have calculate average Transmission Time, Average Propagation Time for each reference node such as R1, R2, R3 to the target node for $7^{\text {th }}$ location.

We have used a reference value as a propagation time to an indexed range. Here we assume,

$$
1 \mathrm{~ns}=1 \text { foot or } 1 \mathrm{~ns}=.3048 \text { meter }
$$


International Journal of Computer Networks \& Communications (IJCNC) Vol.7, No.4, July 2015

From this assumption, we have determined the ranges from each reference node to the target location. Table 3 shows the ranges from each reference node to the target node for its 7 location point.

Table 3: List of the ranges from each reference node to the target node for 7 location point.

\begin{tabular}{|c|c|c|c|c|c|c|}
\hline \multirow{2}{*}{$\begin{array}{c}\text { Target } \\
\text { Location } \\
\text { Point }\end{array}$} & \multicolumn{3}{|c|}{ AWGN Channel } & \multicolumn{3}{|c|}{ Multipath Fading Channel } \\
\hline & $\begin{array}{c}\text { Ref. Node } 1 \\
\text { (m) }\end{array}$ & $\begin{array}{c}\text { Ref. Node } 2 \\
\text { (m) }\end{array}$ & $\begin{array}{c}\text { Ref. Node } 3 \\
\text { (m) }\end{array}$ & $\begin{array}{l}\text { Ref. Node } 1 \\
\text { (m) }\end{array}$ & $\begin{array}{l}\text { Ref. Node } 2 \\
\text { (m) }\end{array}$ & $\begin{array}{c}\text { Ref. Node } 3 \\
\text { (m) }\end{array}$ \\
\hline 1 & 9.2906088 & 9.510677744 & 9.438250147 & 8.92412489 & 9.54115774 & 9.46873015 \\
\hline 2 & 20.671536 & 20.79437374 & 21.03436615 & 20.2440921 & 20.5596777 & 21.0953261 \\
\hline 3 & 43.1983896 & 43.32366575 & 44.34760374 & 43.1976657 & 43.2017457 & 44.3476037 \\
\hline 4 & 82.064352 & 82.97662175 & 83.21082294 & 82.0636281 & 82.9766217 & 83.2108229 \\
\hline 5 & 141.4323816 & 142.0002274 & 141.9558413 & 141.431658 & 142.030707 & 141.955841 \\
\hline 6 & 225.280728 & 225.561757 & 224.9193533 & 225.280004 & 225.561757 & 225.791081 \\
\hline 7 & 337.2852792 & 337.231333 & 337.8047767 & 335.150955 & 335.402533 & 335.671177 \\
\hline
\end{tabular}

After getting all three ranges for a target location, we have computed the average value of three ranges for a location point. Table 4 shows the average value of the ranges for 7 location point.

Table 4: Average range of the target node from three reference node for 7 location point

\begin{tabular}{ccc}
\hline $\begin{array}{c}\text { Target Location } \\
\text { Point }\end{array}$ & $\begin{array}{c}\text { AWGN Channel } \\
\text { Average Range (m) }\end{array}$ & $\begin{array}{c}\text { Multipath Faded Channel } \\
\text { Average Range (m) }\end{array}$ \\
\hline 1 & 9.3113379 & 9.413178897 \\
2 & 20.6330321 & 20.8334253 \\
3 & 43.5823384 & 43.6232197 \\
4 & 82.7503576 & 82.7505989 \\
5 & 141.806069 & 141.7961501 \\
6 & 225.544281 & 225.2539461 \\
7 & 335.408222 & 337.4404629 \\
\hline
\end{tabular}

Then, we have determined the coordinates of the target location by using ranges in multilateration tecnique. In multilateration technique, from each reference node we have drawn a circle where the ranges are used as the radius of the circles. The intersection point of three circles determines the coordinate of the target node. Table 5 shows the final value of X-Y coordinates of the target for 7 location point. According to the value of X-Y coordinate we have plotted a graph in Fig. 4.

Table 5: Value of X-Y coordinates of the target node.

\begin{tabular}{ccccc}
\hline \multirow{2}{*}{$\begin{array}{c}\text { Location } \\
\text { Points } \\
\text { (Number) }\end{array}$} & X-Coordinate & Y-Coordinate & X-Coordinate & Y-Coordinate \\
\cline { 2 - 5 } & 25.79058 & 14.27 & 25.7917 & 14.01 \\
2 & 53.4707 & 30.1006 & 53.58036667 & 30.88736667 \\
3 & 100.1978957 & 57.96786667 & 99.181221 & 56.76436667 \\
4 & 170.1015667 & 97.89706667 & 171.3191667 & 98.01416667 \\
5 & 270.4873667 & 155.9277467 & 271.7887333 & 155.9726333 \\
6 & 406.5874333 & 233.7991333 & 406.6925 & 233.7517 \\
7 & 580.7905333 & 334.6885333 & 581.5436667 & 334.6418 \\
\hline
\end{tabular}




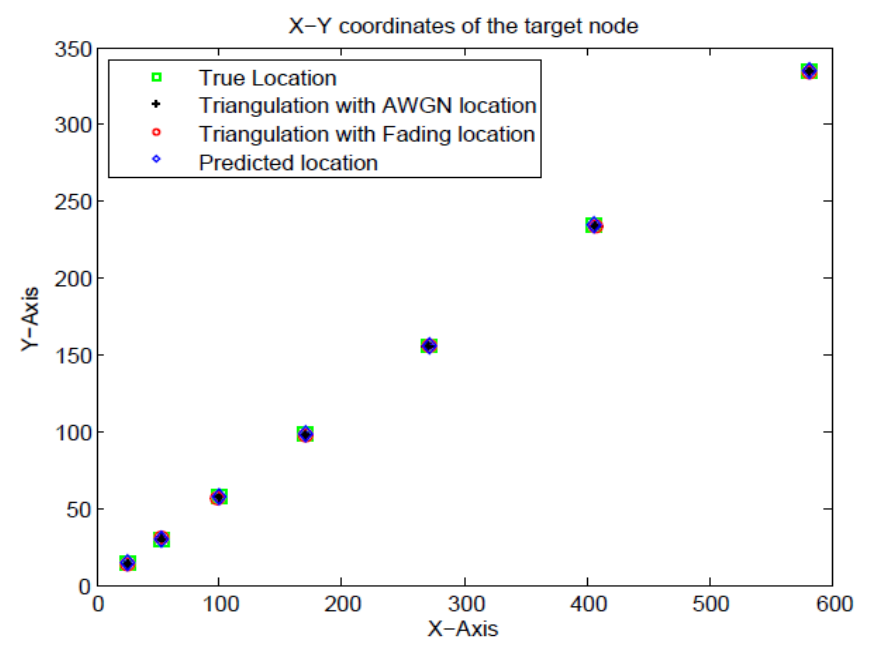

Figure 4: Location Points of the target node.

Figure 4, shows the location points of the target node those are tracked through our implemented PL\&T method under AWGN channel and under fading channel. From Fig.4, we can see that, the location points that we have tracked through PL\&T method under AWGN channel and under fading channel are very close to each other. Thus, it indicates that the developed PL\&T method perfectly handling the effect of multipath fading on tracking accuracy.

\subsubsection{PL\&T of Target Nodes for Spiral Motion}

When a target node moves in a spiral motion, to track the position location of a target node, we have run the simulation for zone finding algorithm and for triangulation algorithm. The simulation process and the result that we have found are discussed below.

Here, we have considered that the target node moves along a spiral path. Since ZF algorithm is based on a polynomial equation, it will predict the position location of the target node along with the straight line. Therefore, when the target node changes its direction ion, through only this $\mathrm{ZF}$ algorithm we cannot predict the position location correctly. For this reason, we need to apply both the ZF algorithm and the triangulation method for tracking the location point accurately. We have followed the same procedure here that we have discussed in section 3.1 to simulate the ZF algorithm.

\section{Simulation of Triangulation after prediction of zone:}

Following the same procedure that we have already discussed in section 4.2.1, we have simulated the triangulation method for find the position location of the target node for its seven location point along the spiral path. From Fig. 5, we have seen that under fading channel, the location points that we have tracked by using triangulation with ZF provide more accuracy than the location points that we have tracked only by zone finding method. 


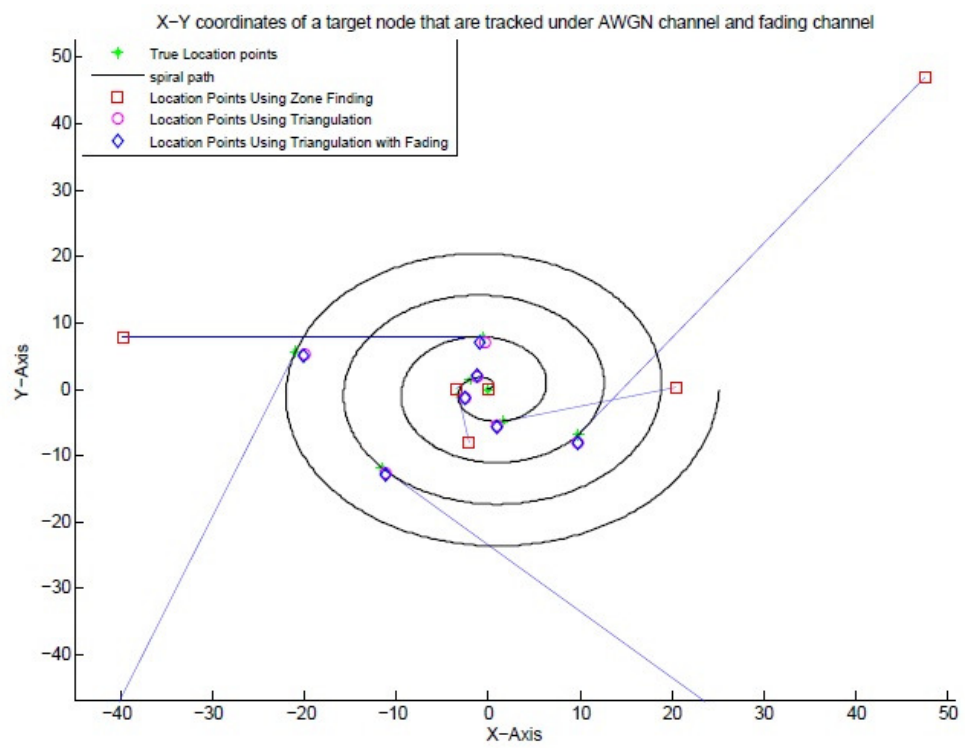

Figure 5: Location points of the target node those are tracked through triangulation method and zone finding method under AWGN channel.

We can see that, in spiral way, the locations those we have found only through the ZF algorithm are far away from the true location points of a target node. Therefore, when target node changes its direction of moving, to track the location points accurately, we need to apply triangulation method with ZF.

\subsection{Performance Analysis:}

We have analysed the performances of implemented PL\&T method by calculating the error between the true value and the result we have found from the real time simulation. For both AWGN channel and Fading channel, using equation (14), we have found out the error of the tracking by measuring the distance between each true location point to the triangulation point of a target node by that is summarized in Table 6 and Table 7 for linear motion and for spiral motion of a target node respectively.

$$
\text { Error in Distance }=a b s \sqrt{\left(x_{\text {trian }}-x_{\text {tmes }}\right)^{2}+\left(y_{\text {trian }}-y_{\text {true }}\right)^{2} \ldots \ldots(14)}
$$

Table 6: Error of Tracking for linear motion of a target node.

\begin{tabular}{ccc}
\hline $\begin{array}{c}\text { Location } \\
\text { points } \\
\text { (Number) }\end{array}$ & $\begin{array}{c}\text { Error of Tracking in AWGN } \\
\text { Channel } \\
(\mathbf{m})\end{array}$ & $\begin{array}{c}\text { Error of Tracking in Rician Fading } \\
\text { Channel } \\
(\mathbf{m})\end{array}$ \\
\hline 1 & $7.14 \mathrm{E}-01$ & $8.36 \mathrm{E}-01$ \\
2 & 0.757075133 & 0.846632406 \\
3 & 0.764783003 & 0.843002755 \\
4 & 0.786762999 & 0.862095678 \\
5 & 0.813777679 & 0.894723049 \\
6 & 0.817858383 & 0.915011099 \\
7 & 0.869564463 & 0.964704045 \\
\hline
\end{tabular}


International Journal of Computer Networks \& Communications (IJCNC) Vol.7, No.4, July 2015

Table 7: Error of tracking for spiral motion of a target node.

\begin{tabular}{ccc}
\hline $\begin{array}{c}\text { Location } \\
\text { points } \\
(\text { Number })\end{array}$ & $\begin{array}{c}\text { Error of Tracking in AWGN } \\
\text { Channel } \\
(\mathbf{m})\end{array}$ & $\begin{array}{c}\text { Error of Tracking in Rician Fading } \\
\text { Channel } \\
(\mathbf{m})\end{array}$ \\
\hline 1 & $8.26 \mathrm{E}-01$ & $9.24 \mathrm{E}-01$ \\
2 & 0.872493837 & 0.94335145 \\
3 & 0.927722647 & 0.978765842 \\
4 & 0.943930312 & 0.99884169 \\
5 & 0.980674617 & 1.035685973 \\
6 & 1.001706679 & 1.091210658 \\
7 & 1.064360996 & 1.15748349 \\
\hline
\end{tabular}

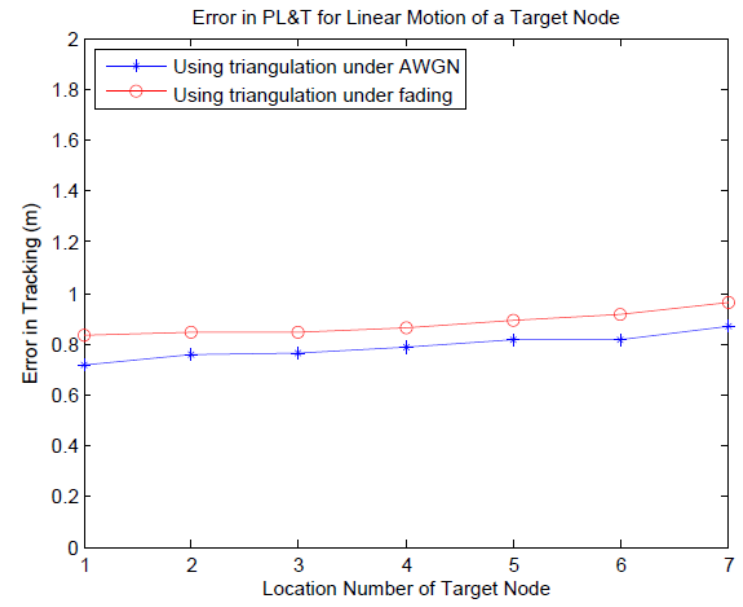

Figure 6: Error of PL\&T under AWGN channel and under fading channel for linear motion.

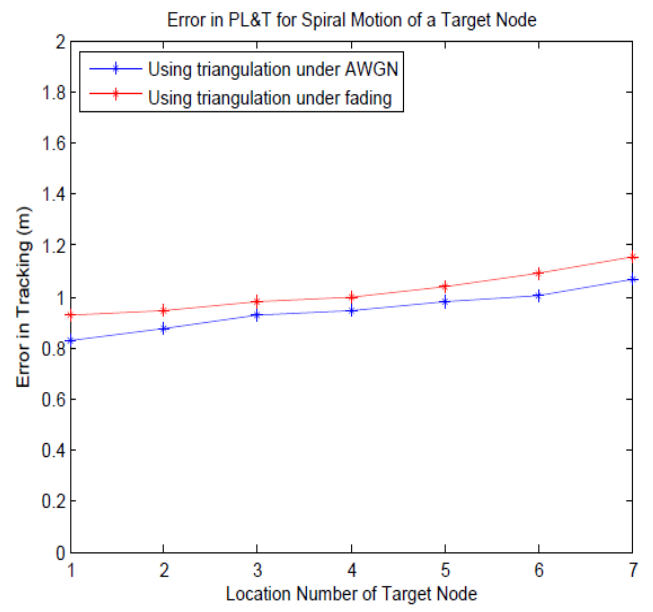

Figure 7: Error of PL\&T under AWGN channel and under fading channel for spiral motion. 
Fig. 6 and Fig. 7 show the graph of the error in terms of the distances for 7 location points of a target node for linear motion and for spiral motion of a target node where X-Axis presents the location number of the target node and Y-Axis presents the distances between each true point and it's tracking points under AWGN channel and under fading channel. We have seen that, the distances between the triangulation points and the true location points under Fading channel are little bit higher than the distances between the triangulation points and the true location points under AWGN channel as we have consider only those transmission delay that have a standard deviation below the threshold value. Therefore, it is shown that the amount of error is very low and it is almost linear for all location points of the target node for both AWGN channel and fading channel. However, it provides more accuracy on tracking under AWGN channel than tracking under fading channel.

We have also analysed the performance of PL\&T by applying different amount of fading during simulation. Fig. 8 shows the graph where we have shown by increasing the amount of fading how it will effect on the tracking accuracy if we consider all the transmission delays. We have used different values for Rician Fading Factor $(\mathrm{K}=1$ to 8$)$ to analyse the impact of fading. In Fig. 8, Xaxis presents the amount of fading in terms of standard deviation of the transmission delay (sigma) under fading channel and Y-axis presents the amount of the error on tracking for a particular location point of a target node under fading channel. We have calculated the standard deviation ( $\operatorname{std}$ or $\sigma$ ) for the transmission delay by using equation (13). We can see that, if we increase the amount of fading, it increases the error of tracking exponentially. Thus, we specify that, if we consider all the transmission delays without measuring the under fading channel, it will reduces the tracking accuracy.

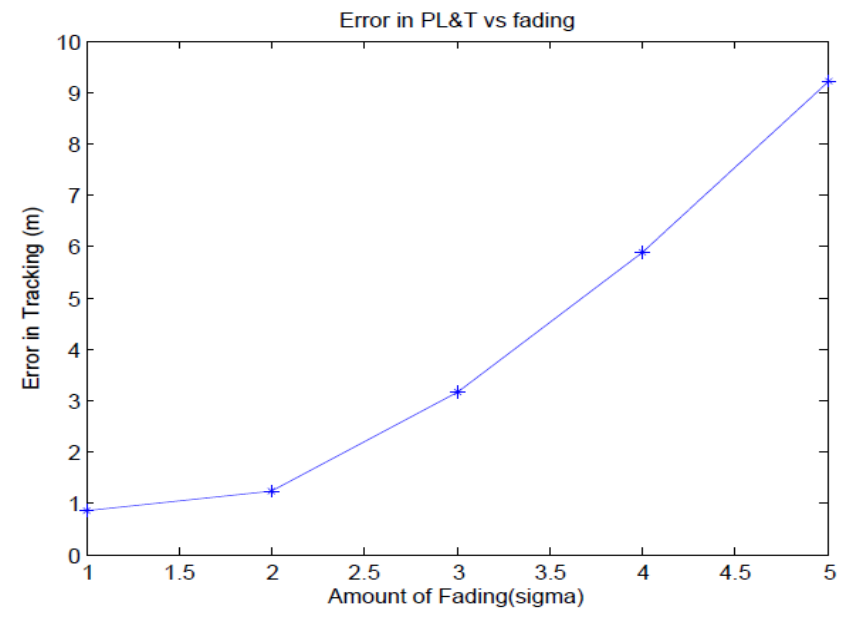

Figure 8: Error in Tracking for different amount of fading.

\section{CONCLUSION}

In this paper, we have analyzed the performance of a real time dynamic Position Location \& Tracking (PL\&T) system that is deployed for MANET using omnidirectional antennas based on zone finding and triangulation method. The integrated zone finding and triangulation based PL\&T system uses zone prediction algorithm which will initially find the zone of a target node, which is used by the triangulation method to place the References to develop proper geometry such that no angle in the formed triangles are less than 30 degrees or greater than 150 degrees. Since both the zone prediction and triangulation method are applied for this PL\&T system, it provides a significant level of accuracy for tracking a mobile object as well as a stationary object. 
Omnidirectional antennas are equipped with nodes to provide efficient ranging and PL\&T operation in MANET. The ranging is done by transmitting an ensemble of IP packets between each reference node and the target node. We determined the error between the true value and the zone predicted value of the target location and error between the true value and the triangulation predicted value of the target location. This is done when using the AWGN channel as well as AWGN plus Multi-Path Fading channel. For the multi-path fading we used Rician Fading channel. Finally, we evaluated the tracking accuracy of the developed PL\&T method for both AWGN channel and fading channel and analyzed the performances through related graphs that are plotted by using real time simulation results. Simulation results show that, in AWGN channel, the distances between the true location points and the location points that we have tracked using this integrated PL\&T system are less than 1 meter for different instances of time. On the other hand, in multipath fading channel, the tracking accuracy is affected slightly due to fading. However, we have considered only the transmission delays of those ensembles of packets that have the standard deviation are below to the threshold value. Thus, in multipath fading channel, the integrated zone finding and triangulation show satisfactory level of tracking accuracy of less than 1.5 meter in both $\mathrm{X}$ and $\mathrm{Y}$ coordinates of the target nodes.

\section{ACKNOWLEDGEMENT}

This work is supported in part by the US Army Research Office under the Research Cooperative Agreement Grant, W911NF-04-2-0054 to the ARO Center for Battlefield Communications (CeBCom), National Science Foundation under the contract 0931679 and 1229744, Department of Electrical and Computer Engineering, Prairie View A\&M University (Texas A\&M University System). The views and conclusions contained in this dissertation are those of the author and should not be interpreted as representing the official policies, either expressed or implied, of the Army Research Office or the U.S. Government.

\section{REFERENCES:}

[1] N. Shakhakarmi, D. R. Vaman, "Real Time Position Location \& Tracking (PL\&T) using Prediction Filter and Integrated Zone Finding in OFDM Channel", WSEAS transactions in Communications, Vol. 11, July 2012.

[2] M. Porretta , P. Nepa, G. Manara, F. Giannetti , "Location,Location,Location", Vehicular Technology Magazine, IEEE,Vol.3, pp 20-29, June 2008

[3] S. Khan, D. R. Vaman, S. T. Koay, "Highly Reliable Multi-Service Provisioning Using Sequential Prediction of Zone and Pl\&T of Nodes In Mobile Networks", International Journal of Wireless \& Mobile Networks (IJWMN), Vol. 6, No. 4, August, 2014

[4] L. Xiaofeng, W. Fletcher, L. Ian, L. Pietro, X. Zhang, "Location Prediction Algorithm for Directional Communication", Computer Laboratory, University of Cambridge, U.K, Beijing, University of Aeronautics and Astronautics Beijing, UK, IWCMC, 2008.

[5] N. Shakhakarmi, D. R. Vaman, "Dynamic PL\&T using Two Reference Nodes Equipped with Steered Directional Antenna for Significant PL\&T Accuracy", Wireless Telecommunications Symposium 2012, London, UK.

[6] N. Shakhakarmi, D. R. Vaman, "Distributed Position Localization and Tracking (DPLT) of Malicious Nodes in Cluster Based Mobile Ad hoc Networks (MANET)", WSEAS transactions in Communications, Vol. 9, November 2010.

[7] L. Xiaofeng, W. Fletcher, L. Ian, L. Pietro, X. Zhang, "Location Prediction Algorithm for Directional Communication", Computer Laboratory, University of Cambridge, U.K, and College of Computer Science, Beijing University of Aeronautics and Astronautics, China, IWCMC, 2008.

[8] K. Kathiravan, S. S. Thamarai, "An Efficient Location Tracking Algorithm for MANET using Directional Antennas", Signal Processing, Communications and Networking, 2007. ICSCN '07. International Conference on , pp 139-144, February,2007 
International Journal of Computer Networks \& Communications (IJCNC) Vol.7, No.4, July 2015

[9] R. Siuli, C. Sanjay, B. Somprakash, U. Tetsuro, I. Hisato \& O. Sadao, (2005) "Neighborhood Tracking and Location Estimation of Nodes in Ad hoc Networks Using Directional Antenna: A Testbed Implementation", Proceedings of the Wireless Communications Conference, Maui, Hawaii, USA.

[10] B. Zhang, F. Yu, "Low-complex energy-efficient localization algorithm for wireless sensor networks using directional antenna," Department of Integrated Electronics, Shenzhen Institutes of Advanced Technology, IET Commun., 2010, Vol. 4, pp. 1617-1623, 2010

[11] N. Shakhakarmi, D. R. Vaman, "Dynamic Position Location and Tracking using Location based Hash Scheme for Malicious Detection under Doppler Spread Rayleigh Channel”, Submitted to IEEE Transactions on Wireless Communications, October, 2011

[12] MQ Brewster, KT Wang, WH Wu, MG Khan, "Temperature effect on phase-transition radiation of water", Journal of Heat Transfer, 2014, Vol. 136(6), 062704, doi: 10.1115/1.4026556

[13] P.Veeranath, D.N.Rao, S.Vathsal, N.Bhasker," Reducing Multipath Effects in Indoor Channel for Analysis of GPS/Pseudolite Signal Acquisition", International Journal of Scientific and Research Publications, Vol.3, February 2013, url:" www.ijsrp.org".

[14] Estimation and mitigation of gps multipath interference using adaptive filtering,Progress In Electromagnetics Research M, Vol. 21,

[15] G.R. Khan, D.R. Vaman, "Modeling and Designing Resource Efficient Distributed MANET Management System (DMMS)", International journal of Computer Networks \& Communications, Vol.6, No. 5, September. 2014.

[16] G.R. Khan, S. Khan, D. R. Vaman, S. Cui, "A Novel Resource Efficient DMMS Approach for Network Monitoring and Controlling Functions", International Journal of Wireless Mobile Networks (IJWMN), Vol. 7, No. 1, February 2015.

[17] R. Ramanathan ,Antenna Beamforming and Power Control for Ad Hoc Networks, , BBN Technologies, Cambridge, Massachusetts, url:" http://www.ir.bbn.com/ ramanath/pdf/wileybookchap.pdf".

[18] R. Ramanathan, J. Redi, C. Santivanez, D. Wiggins, S. Polit, "Ad Hoc Networking with Directional Antennas: A Complete System Solution”, IEEE J. Sel. Areas Commun., Vol. 23, pp. 496-506, March 2005.

[19] Gilbert, J.M. ; Atheros Commications, Inc., Sunnyvale, CA, USA ; Won-Joon Choi ; Qinfang Sun," MIMO Technology for Advanced Wireless Local Area Networks",Design Automation Conference, 2005. Proceedings. 42nd, pp.413 - 415, June 2005

[20] Omnidirectional vs Directional, url "http://www.cisco.com/c/en/us/support/docs/ wirelessmobility/wireless-lan-wlan/82068-omni-vs-direct.html".

[21] G. R. Khan, “ Coded Cooperative Diversity with Turbo Codes Over Wireless Fading Channels ”, MS Thesis, Tuskegee University, Tuskegee Institute, AL, USA, December, 2010

[22] S. Kumar, M. G. Sumithra, M. Sarumathi, "Performance Analysis of Rician Fading Channels using Non-linear Modulation Methods with Memory Schemes in Simulink environment", IOSR Journal of Computer Engineering, vol.11, pp.27-36, June, 2013

[23] Jean, Paul, M.G. Linnartz, "Rician Fading", Wireless Channels, Multipath Fading in JPL's Wireless Communication Reference Website, internet: http://www.wirelesscommunication.nl/ reference/chaptr03/ricepdf/rice.htm

[24 ]A. Doukas, G. Kalivas, "Rician K Factor Estimation for Wireless Communication Systems", in Proceding on International Conference on Wireless and Mobile Communications, 2006. ICWMC '06, IEEE, pp. 69, July, 2006.

\section{Authors}

Sharmistha Khan got the B.Sc. degree in Computer Science from American International UniversityBangladesh (AIUB), Dhaka, Bangladesh in 2006, and the M.S. degree in Electrical Engineering from Tuskegee University, Tuskegee, AL in 2011, respectively. She is currently completed her Ph.D degree in the department of Electrical and Computer Engineering at the Prairie View A \& M University, Prairie View, TX. She was working under the supervision of Prof. Dhadesugoor R. Vaman and Prof. Siew T. Koay at Prairie View A \& M University. Her research area includes Mobile Adhoc Network, Cognitive Radio Networks, Sensor Networks, Mobile WiMAX Technology, Handoff Management, and Handoff Performance and Decision Making Algorithms for Broadband Wireless Networks. She is a member of the CEBCOM group at the Prairie View A \& M University. 
Golam R. Khan has completed his Ph.D degree from the department of Electrical Engineering of Prairie View A\&M University (PVAMU), TX. Previously, he was working as a Graduate Research Assistant in the Center of Battlefield Communication (CeBCom). His advisors are Prof. Dhadesugoor R. Vaman and Dr. Suxia Cui. His current research interest is "Modeling and designing resource efficient Distributed MANET Management System (DMMS) for multi-service applications". He got his Bachelor of Science degree in Computer Science (CS) from American International University Bangladesh (AIUB), Bangladesh in May 2006. After his B.Sc, he worked in software industries for 2 years. He also completed his Master's degree in Electrical Engineering (EE) from Tuskegee University (TU), AL in December 2010. In his Master's thesis, he worked with 'Coded Cooperative Diversity with Turbo Codes' and his advisor was Dr. Fan Jiang.

Dhadesugoor R. Vaman is Texas Instrument Endowed Chair Professor and Founding Director of ARO Center for Battlefield Communications (CeBCom) Research, ECE Department, Prairie View A\&M University (PVAMU). He has more than 38 years of research experience in telecommunications and networking area. Currently, he has been working on the control based mobile ad hoc and sensor networks with emphasis on achieving bandwidth efficiency using KV transform coding; integrated power control, scheduling and routing in cluster based network architecture; QoS assurance for multi-service applications; and efficient network management. Prior to joining PVAMU, Dr. Vaman was the CEO of Megaxess (now restructured as MXC) which developed a business ISP product to offer differentiated QoS assured multiservices with dynamic bandwidth management and successfully deployed in several ISPs. Prior to being a CEO, Dr. Vaman was a Professor of EECS and founding Director of Advanced Telecommunications Institute, Stevens Institute of Technology (1984-1998); Member, Technology Staff in COMSAT (Currently Lockheed Martin) Laboratories (1981-84) and Network Analysis Corporation (CONTEL)(1979-81); Research Associate in Communications Laboratory, The City College of New York (1974-79); and Systems Engineer in Space Applications Center (Indian Space Research Organization) (1971-1974). He was also the Chairman of IEEE 802.9 ISLAN Standards Committee and made numerous technical contributions and produced 4 standards. Dr. Vaman has published over 200 papers in journals and conferences; widely lectured nationally and internationally; has been a key note speaker in many IEEE and other conferences, and industry forums. He has received numerous awards and patents, and many of his innovations have been successfully transferred to industry for developing commercial products.

Suxia Cui is an associate professor in the Department of Electrical and Computer Engineering at Prairie View A\&M University (PVAMU). She received her BS and MS degrees both in Electrical Engineering from Beijing University of Technology in 1996 and 1999 respectively. Right after obtained her PhD degree in Computer Engineering from Mississippi State University in 2003, Dr. Cui joined Prairie View A\&M University. Her research interests include image and video processing, data compression, wavelets, computer vision, remote sensing, and computing education. She has published numerous papers, and her research is funded by United States Department of Agriculture, Department of Education and National Science Foundation. 